\title{
Recent Scientific Breakthroughs Applying CRISPR Gene Editing in Neurological Disorders
}

\author{
Mitra Assadi-Khansari, MD, FAAN and London Pamela McGill, MS
}

Over the past three decades, technological advances have revolutionized the diagnosis and management of neurological disorders. No longer mystifying or ambiguous, even the most uncommon afflictions of the nervous system are now easily defined by molecular markers. The opportunity to recognize incurable disorders often puts neurologists in the juxtaposition of having to deliver bad news without any remedies.

Genome editing technology offers a great opportunity for treating numerous fatal neurological ailments caused by genetic etiologies. The journey in gene editing begins with recognizing the faulty gene causing the disorder, followed by disrupting the gene sequence using a very precise tool called CRISPR/Cas (l) CRISPR-associated). Cas comprises a family of nucleases synthetized by bacteria as part of their adaptive immunity against viruses, of which Cas9 (CRISPR-associated protein 9) is the most commonly utilized version for gene editing (see Figure 1). ${ }^{1,2}$

Figure 1: CRISPR Associated Nucleases (Cas) Briefly Explained

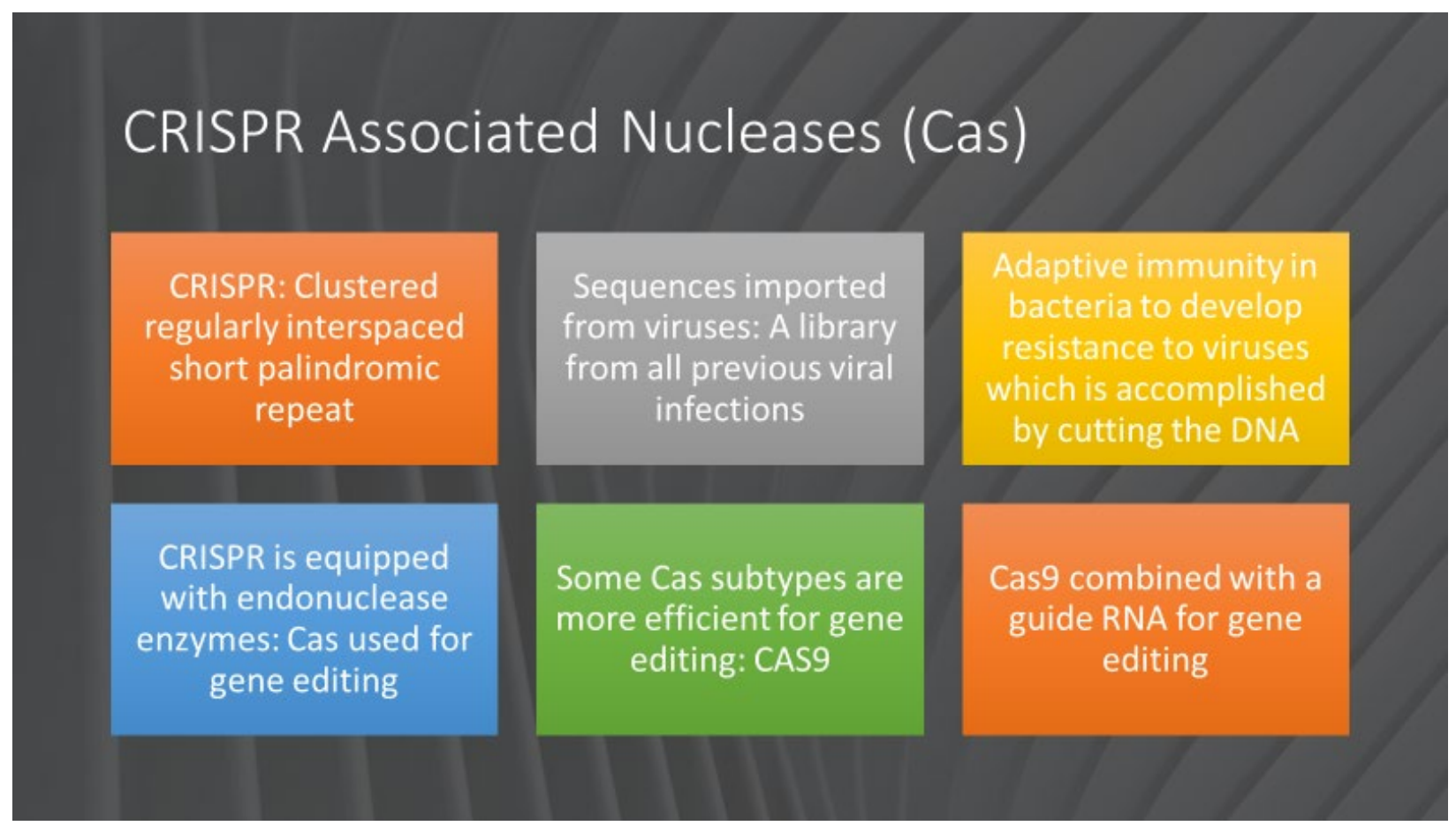

The CRISPR/Cas system works by using short RNA sequences known as guide RNA's (gRNA's) to recognize the affected allele(s). This 20-nucleotide sequence follows a PAM (Protospacer Adjacent Motif) sequence which helps to ensure site specific binding and cleavage. If the target PAM site has been disrupted or if the gRNA sequence has more than six nucleotide mismatches with the target site, the gRNA will not bind and the CRISPR cannot perform the cleavage at that site. ${ }^{2,3}$ The gRNA acts as a guide for the Cas protein, directing it to the target site, where it then cleaves the DNA creating a double stranded break three base-pairs upstream from the PAM site. Once the break is created one of two repair mechanisms can be utilized. ${ }^{4}$ 
The "knock out" model utilizes the two double stranded breaks which are created upstream from one another and the DNA is left to repair itself by recombining the blunt ends of DNA. This method is often used to remove codons and shift the open reading frame (ORF) out of frame resulting in a "knockout" of the gene of interest.

The second method, the "knock in" model, uses a single gRNA to open the DNA at the point of interest and a repair template matching the DNA sequence, giving the DNA a blueprint to copy during repair. Figure 2 depicts the process of the CRISPR/Cas complex as it creates a double stranded break promoting either NHEJ or HDR to either create a desired indel (knockout) or create a precise correction such as a knock in or point mutation repair. ${ }^{4}$ The knock in model introduces new genetic material to correct the point mutations or frameshift mutations.

Figure 2: CRISPR Induced Cell Repair

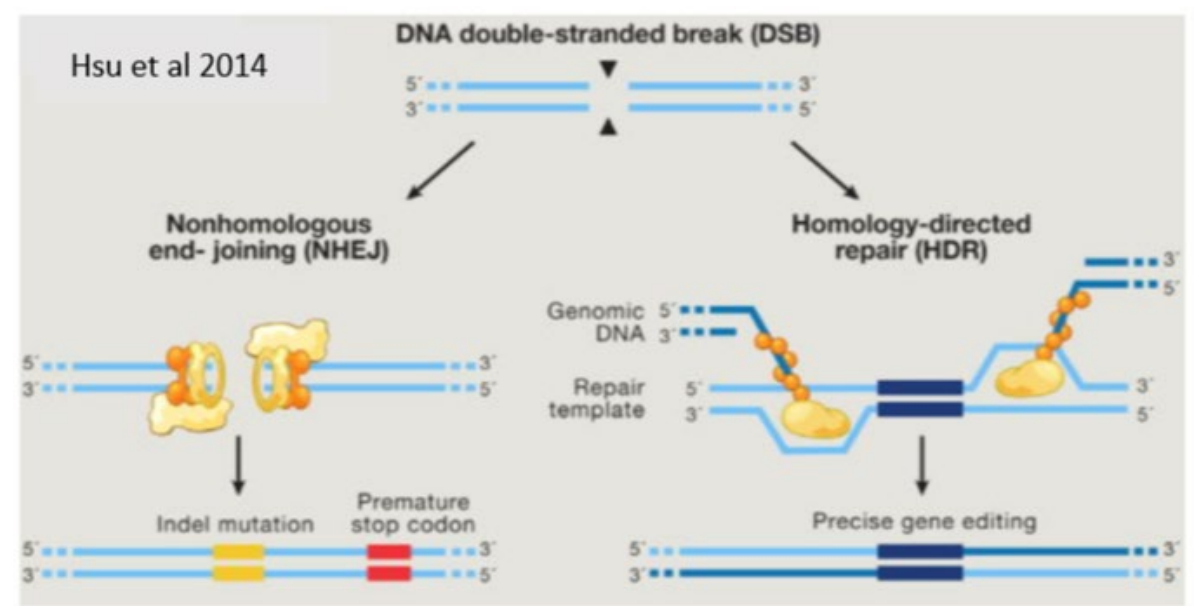

CRISPR technology can be implemented both in-vitro and in-vivo. Figure 3 highlights the steps needed to complete in vitro gene editing for in vivo use. Mesenchymal stem cells are harvested from adipose tissue, they then undergo gene editing and are expanded in vitro until there are a suitable number of cells to be injected back into the human or mouse model. ${ }^{1}$ The in-vitro model involves harvesting and editing cells, expanding them in a cell culture and delivering them back to the subject. Stem cell interventions exemplify a major application for CRISPR technology. For instance, for treating ALS or repairing spinal cord injuries, mesenchymal stem cells may be harvested from the adipose tissue, edited, and then delivered intrathecally. Autologous stem cell interventions are used for treating numerous otherwise untreatable neurological conditions such as leukodystrophies. ${ }^{1,5}$

Figure 3: Stem Cell Interventions in Neurological Disease 


\section{Stem cell interventions}
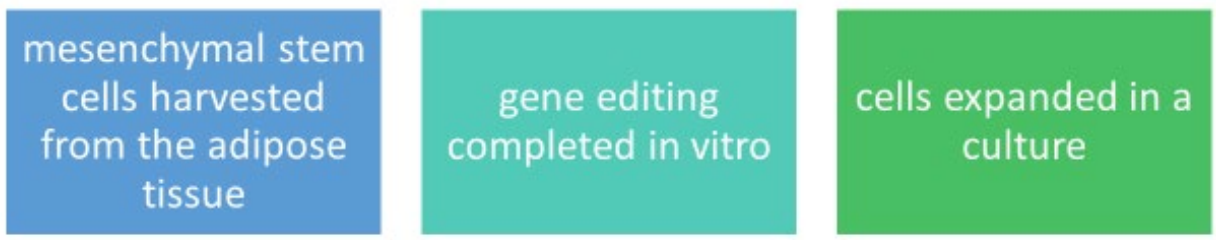

Injection to the

animal model or

human subject

Other examples of vectors include liposomes or synthetic nanoparticles. These vectors are driven to the cell membranes due to their lipid solubility and as such, enhance the delivery of the gene editing complex. ${ }^{6}$

Neurodegenerative disorders comprise numerous devastating and often fatal conditions which are a major source of social and economic burden. Figure 4 highlights five common neurological disorders, the genetic cause, and how the disease can be corrected using CRISPR/Cas based therapeutics..$^{5-7}$ CRISPR-Cas9 has been used to create cell and animal models of the diseases, as well as to target the genes to mitigate the abnormality. The scientific community is intrigued by the progress being made in applying gene editing in a variety of neurodegenerative disorders. These strides begin by correcting the molecular abnormality at the cellular level using CRISPRCas9 before applying the intervention to animal models. ${ }^{5,8}$ The recent application of gene editing in six human subjects with amyloidosis caused by transthyretin mutation demonstrated its safety and efficacy in decreasing the abnormal protein level. ${ }^{7}$

Figure 4: Applications of Gene Editing in Degenerative Disorders 
Doi: $10.32481 /$ djph.2021.12.004

Applications of gene editing in degenerative disorders

\begin{tabular}{|c|c|c|c|}
\hline Disease & genetic loci & in vitro application & in vivo application \\
\hline Alzheimer's disease & APP, PSEN1, PSEN2 & iPSC derived neurons & $\begin{array}{c}\text { CRISPR/AAV } \\
\text { intraparenchymal } \\
\text { injection, transgenic mice }\end{array}$ \\
\hline Frontotemporal dementia & C9ORF, MAPT & Knockout model & in progress \\
\hline Huntington disease & Huntingtin gene & Fertilized oocytes & $\begin{array}{c}\text { CRISPR/AAV } \\
\text { intraparenchymal } \\
\text { injection, mice/monkey }\end{array}$ \\
\hline ALS & SOD, C9ORF & Mesenchymal stem cells & intrathecal injection \\
\hline $\begin{array}{l}\text { Duchenne muscular } \\
\text { dystrophy }\end{array}$ & dystrophingene & Exon 44 skipping & $\begin{array}{l}\text { IV/IM Injection in the } \\
\text { mouse model }\end{array}$ \\
\hline
\end{tabular}

The major challenges in gene editing include limited efficiency and precision. While scientists hope to target at least $50 \%$ of the mutated alleles to rectify the effects of the faulty gene, even in the best cases, the CRISPR-Cas complex edits less than $10 \%$ of the alleles. Moreover, due to partial matches between the gRNA and other parts of the genome, off target effects occur frequently. The PAM mechanism helps to ensure that off-target activity does not occur when making corrections to mutant alleles. The gene editing field continues to work on correcting off target effects as well as further studying the CRISPR/Cas system to make it a more predictable system for therapeutic use.

It is important to note that other viral vectors are available with the larger packaging capacity needed to carry the CRISPR/Cas system in-vivo. Many of these other vectors such as lentivirus, retrovirus, and even some species of AAV come with the risks however, such as random incorporation into the host genome at unintended sites which can lead to dysregulation of nearby genes, chromosomal rearrangement, or even genotoxicity in some tissue types, as well as unstudied effects on brain homeostasis. ${ }^{9,10}$ Although unintended incorporation of certain AAV vectors is not commonly seen in neurological tissue and disease studies it is still something that should be monitored as new vectors are developed and introduced into the field.

In summary, gene editing is a powerful and promising tool which will revolutionize the treatment of neurogenetic disorders, and given the rapid advances made over the past few years, is expected to come to fruition during our professional lifetime. We aim to educate the providers in our community regarding the existing alternatives and the ongoing research in this field.

These authors can be contacted at london.mcgill@,christianacare.org

\section{References}

1. Madigan, N. N., Staff, N. P., Windebank, A. J., \& Benarroch, E. E. (2017, October 17). Genome editing technologies and their potential to treat neurologic disease. Neurology, 89(16), 1739-1748. PubMed https://doi.org/10.1212/WNL.0000000000004558

2. Jinek, M., Chylinski, K., Fonfara, I., Hauer, M., Doudna, J. A., \& Charpentier, E. (2012, August 17). A programmable dual-RNA-guided DNA endonuclease in adaptive bacterial immunity. Science, 337(6096), 816-821. PubMed https://doi.org/10.1126/science.1225829 
3. Collias, D., \& Beisel, C. L. (2021, January 22). CRISPR technologies and the search for the PAM-free nuclease. Nature Communications, 12(1), 555. PubMed https://doi.org/10.1038/s41467-020-20633-y

4. Hsu, P. D., Lander, E. S., \& Zhang, F. (2014, June 5). Development and applications of CRISPR-Cas9 for genome engineering. Cell, 157(6), 1262-1278. PubMed https://doi.org/10.1016/j.cell.2014.05.010

5. Kuruvilla, J., Sasmita, A. O., \& Ling, A. P. K. (2018, November). Therapeutic potential of combined viral transduction and CRISPR/Cas9 gene editing in treating neurodegenerative diseases. Neurol Sci, 39(11), 1827-1835. PubMed https://doi.org/10.1007/s10072-018$\underline{3521-0}$

6. Barman, N. C., Khan, N. M., Islam, M., Nain, Z., Roy, R. K., Haque, A., \& Barman, S. K. (2020, December). CRISPR-Cas9: A promising genome editing therapeutic tool for Alzheimer's disease-A narrative review. Neurology and Therapy, 9(2), 419-434. PubMed https://doi.org/10.1007/s40120-020-00218-z

7. Gillmore, J. D., Gane, E., Taubel, J., Kao, J., Fontana, M., Maitland, M. L., . . Lebwohl, D. (2021, August 5). CRISPR-Cas9 in vivo gene editing for transthyretin amyloidosis. The New England Journal of Medicine, 385(6), 493-502. PubMed https://doi.org/10.1056/NEJMoa2107454

8. Min, Y. L., Li, H., Rodriguez-Caycedo, C., Mireault, A. A., Huang, J., Shelton, J. M., .. . Olson, E. N. (2019, March 6). CRISPR-Cas9 corrects Duchenne muscular dystrophy exon 44 deletion mutations in mice and human cells. Science Advances, 5(3), eaav4324. PubMed https://doi.org/10.1126/sciadv.aav4324

9. Tosolini, A. P., \& Sleigh, J. N. (2020, July 17). Intramuscular delivery of gene therapy for targeting the nervous system. Frontiers in Molecular Neuroscience, 13, 129. PubMed https://doi.org/10.3389/fnmol.2020.00129

10. Deyle, D. R., \& Russell, D. W. (2009, August). Adeno-associated virus vector integration. Current Opinion in Molecular Therapeutics, 11(4), 442-447. PubMed

Copyright (c) 2021 Delaware Academy of Medicine / Delaware Public Health Association.

This is an Open Access article distributed under the terms of the Creative Commons Attribution Non-Commercial License (https://creativecommons.org/licenses/by-nc-nd/4.0/) which permits unrestricted non-commercial use, distribution, and reproduction in any medium, provided the original work is properly cited. 